\title{
In Search of Valid Results in a Complex Economic Environment: The Potential of Meta-analysis and

\author{
Value Transfer
}

\author{
October 1997 \\ Frans Bal and Peter Nijkamp \\ Free University of Amsterdam \\ Dept. of Spatial Economics and Tinbergen Institute \\ De Boelelaan 1105 \\ NL-1081 HV Amsterdam \\ The Netherlands
}

\begin{abstract}
The rapidly increasing dissemination of scientific results exhibits features of complex systems and brings also to light (seemingly) inconsistencies among research findings. At the same time, there is a fast growing interest in comparative studies and related research tools such as metaanalysis and value transfer. Recently, several studies have been published in the economic literature with the aim to test the performance of value transfer in a direct way. The results from previously independently undertaken studies are then compared with the results generated by value transfer: one of the independent studies is chosen as a study site and serves as a benchmark for value transfer, while the other site is treated as the policy site. Distinct studies show that the values obtained for the policy site via value transfer do not necessarily comply with the results obtained from the independently undertaken study at that site. This paper will offer a critical methodological contribution to the discovery of sources of difficulties underlying the application of value transfer and the creation of new knowledge. The paper offers new insights by considering value transfer in the light of modern quantitative economic analysis as well as a formal framework for the applicability of value transfer in economic research. Throughout the paper, simple cases will serve as illustrations for our arguments.
\end{abstract}




\section{Introduction}

In our age of informatization, an exponentially growing number of studies is being published. The quantitative revolution in the social sciences - and in economics in particular - has generated an avalanche of new models and new empirical findings. Any overview of dedicated publications on a certain theme will show that a (partial) repetition of studies concerning the subject concerned is not uncommon. An important reason to repeat - partly or entirely - an existing study is the fact that there are often conflicting outcomes over a wide range of independently undertaken studies. Especially in case of complex (nonlinear dynamic) systems unexpected differences in system's outcomes may occur. Of course, such a seemingly inconsistency in empirical scientific results may question the validity of the research undertaken. The most important consequence of such conflicting results is that a theory concerning a certain issue does apparently not lead to conclusive answers. The application of different analytical frameworks or different data bases with the aim of investigating similar phenomena may turn out to exhibit a complex array of varying results. When we consider inconsistencies, we may also wonder how robust empirical findings from quantitative economic studies in an ever changing external and complex environment are. In other words: may scientific findings be claimed to have a wider applicability than the case studies they were derived from?

This is a fascinating questing which may essentially be interpreted in terms of complex systems analysis. A complex system may exhibit various types of behaviour (stable, chaotic etc.) depending on initial conditions, the external environment and indigenous system's (or actors') behaviour. In the same vein, a certain phenomenon (e.g. price elasticity of some good) may in various scientific studies exhibit a wide range of values, depending on the site-specific conditions (initial conditions), the overall economic development (the external environment) and the actors' response to some policy intervention (the indigenous behaviour). Comparing the results of such studies is essentially nothing else than complex system analysis, at either the level of numerical outcomes or at the level of decisions taken (see also De Tombe, 1994).

In the past decade, several methodological advances have been made to separate out the various causes of conflicting results (e.g., site-specific elements, data uncertainty, methodological differences etc.). Especially in meta-analysis - and to some extent also value (or benefit) transfer - a systematic framework has been developed for distinguishing between various classes of sources of differences in outcomes. Meta-analysis aims to provide a quantitative synthesis of scientific results from studies on a similar issue, while value transfer seeks to develop a framework for transferability of value (or benefit) estimates of policy decisions.

Beside a growing interest in dealing with conflicting scientific results, currently many efforts are undertaken to carry out comparative studies which make use of meta-analysis and/or value transfer. In particular in environmental economic research, a direct test on value transfer by conducting two parallel case-studies with the aim to derive non-market values and to compare these with the results obtained via value transfer has been undertaken by several researchers; see Bergland et al. (1995), Kirchhoff et al. (1997) and Bateman et al. (1995). Beside the casestudy approach adopted inter alia by Bergland et al. (1995) to derive the required values for value transfer purposes, also meta-analysis can be used to obtain estimations of non-market values. In this context we refer also to e.g. Waters (1995), Button and Kerr (1996) and Button 
and Rietveld (1997).

However, several studies on value transfer do not show very promising results. This questions the validity of this research tool. In this paper we will consider inconsistencies in (case-) study findings, seen from the perspective of meta-analysis and value transfer applications. By a meta-level study (see Matarazzo and Nijkamp, 1997) we aim to address various problems induced by the analytical complexity of the real world, its impact on research findings, the potential of meta-analysis to deal with these problems, and the way it may affect the performance of value transfer.

Clearly, any attempt to cope with a situation of conflicting results will start with some form of critical review of existing results in order to discover the sources yielding the inconsistency with the aim to reformulate the problem definition and hence to complete the theory concerned. In the light of the previous observations this paper is organized as follows. Section 2 will take a closer look at various inconsistencies among scientific results and at the question how meta-analysis approaches can be used to overcome these. In Section 3 a systematic overview of the main methodological differences between meta-analysis and value transfer will be presented. After a discussion in Section 4 of the various techniques which generate knowledge appropriate for value transfer, the domain of application of value transfer will be outlined. Section 5 then proceeds by considering the theoretical core which also points out the limitations of transferability of knowledge, while the implications for the overall potential of value transfer will be analyzed in Section 6. Concluding remarks are offered in the final section. Throughout the paper, simple illustrative examples will mirror the practical sides of the theoretical/methodological basis presented here.

\section{Meta-analysis as a Method of Study}

In scientific research experiments, it is a matter of research efficiency to separate the specific from the general, the novel from the well-known results. Case studies mainly aim at generating new knowledge, and do not serve to confirm existing knowledge through a repetition of experiments. The reason for a repetition of a study is for a significant part caused by observed or expected conflicting outcomes over a range of independent studies undertaken in the past on a certain issue. Methodologically, such conflicting outcomes may stem from differences in research methods, analytical techniques, theoretical assumptions, data bases and so on; we refer to Parsons and Kealy (1994) and Carson et al. (1996) for a more detailed treatment. In this framework, it is interesting to observe that Van den Bergh and Button (1997) recognize that, when the results of a Contingent Valuation method (further abbreviated to $\mathrm{CV}$ method) are compared with those of a Revealed Preference method, the qualitative conclusions may be identical, even though the quantitative indicators differ in value.

Of course, inconsistency in results, mostly obtained on the basis of different empirical studies, provokes intriguing methodological questions which need to be resolved to make theory-building possible, especially in the social sciences where a tightly controlled experiment is most of the time absent. Inconsistency is in this situation an intriguing problem, since usually no fully controlled experiment can be designed and implemented to generate a final and conclusive answer. In fact, the background of most efforts in scientific case study research is 
that via empirical studies new knowledge is stepwise accumulated with the aim to create new explanatory theories - or to improve incrementally the existing theories - on a certain theme. When quantitative empirical results lead to seemingly contradictory inferences, an explanatory theory concerning a certain issue cannot unambiguously be developed. Clearly, in such a situation observed results should carefully be reviewed to discover the sources yielding the inconsistency and to describe a revised (improved) problem definition for further research with the aim to complete the theory. Therefore, it is necessary to identify the methodological backgrounds leading to inconsistent results across studies undertaken thus far. The causes that may lead to inconsistent results are diverse in character. To mention some: (a) an unsatisfactory handling of the cumulated information within the initiated research project; (b) an unreliable data collection or observation process; (c) an improper use of methods of analysis within the methodological framework; (d) an inappropriate methodological framework within which the applied research has taken place.

The errors caused by (a) and (b) are straightforward. Errors due to (c) and (d) are less clearly present or identified in research practice. Against this background, we may refer to Gowdy (1997) who argues that, when biodiversity is investigated in the traditional neoclassical methodological framework, fundamental shortcomings are present. More methodological difficulties were shown by Sagoff (1988) who analyzed the intended investments by Walt Disney within the Mineral King Valley in California. In this context, the author found a fabulous difference between the choices people apparently made as consumers and choices they made as citizens.

Another major source of inconsistency in empirical findings in the social sciences has usually a statistical origin. From a quantitative perspective, studies undertaken in the social sciences may suffer from three errors which can be classified as important potential sources of inconsistency: sampling errors; measurement errors; and range variations.

These errors will now concisely be illustrated. All these three sources of error stem from empirically obtained knowledge, which is the major source of information for the social sciences. This information is a necessity for analysis and synthesis (building theories) in these sciences. In an interesting (theoretical) example, Hunter and Schmidt (1990) show how inconsistent results among different "studies" can be related to a sampling error. In their artificial example, the observed explanatory effects are the result of chance. In reality, it is not unlikely that such a situation is present in many empirical studies. Besides this type of error on the level of data collection, errors may also emerge due to data handling such as the use of different measurement scales and round-off errors. These statistical errors are in practice disturbing for scientific research in general and for meta-analysis - as a procedure for a systematic synthesis of empirical results - in particular. Invalid results lead, when they remain undiscovered, to unjustified research findings and, as a logical consequence, to inconsistent conclusions on the initial study level as well as on the meta-level (see Matarazzo and Nijkamp, 1997).

Social science research appears to be fraught with many statistical uncertainties. In the history of the social sciences, the significance test is most often used to test hypotheses. Hunter and Schmidt (1990) correctly point out that when the error rate is equal to a and the null hypothesis is false, the error rate can fall between á and (1-á), with á $\in[0,1]$. To reduce the 
emergence of this potential problem underlying the test of statistical significance, the authors suggest two alternatives:

$\square$ for single studies, the use of confidence intervals, and

$\square$ for review studies, the use of meta-analysis.

Meta-analysis is one of the techniques which generates synthetic knowledge useful for value transfer. In the sequel of this paper we will in particular address meta-analytic approaches (see Van den Bergh et al., 1997) where statistical techniques can be used, thereby neglecting the broad spectrum of other meta-techniques available to synthesize over a wide collection of studies. According to Van den Bergh et al. (1997), nowadays meta-analysis does not only leave room for the application of purely quantitative techniques for synthesing research outcomes. In fact, the format of the data and the objective of the synthesis will determine the technique used to arrive at a synthesis, given a well-defined collection of independent, but similar studies. Clearly, to determine the functional form and the parameter values between variables as well as to discover evolving patterns, the use of suitable statistical techniques such as regression is straightforward. For a qualitative analysis however, a comparative evaluation or ranking of individual studies can be used. Finally, when single studies are to be grouped and classified on numerical characteristics which are not necessarily precisely measured, rough set analysis is probably the most suitable technique for synthesis. Returning to a form of metaanalysis techniques where only statistical techniques are used, it is obvious that when a regression is carried out on a data set - in which each study is treated as a single data source which suffers from statistical errors, the correlation coefficient obtained is subject to this source of error as well. However, meta-analysis has the interesting potential to correct for statistical errors on this very same level. As Hunter and Schmidt (1990) show, it is possible to test a well-defined collection of studies on the presence of variance that stems from a sampling error via meta-analysis. Basically, these problems which are due to this artificial error, can be overcome by correcting the mean and the variance. Then, a new regression can be carried out among the initial well-defined collection of studies corrected for statistical errors.

Given the data set corrected for statistical errors, a meta-regression is able to show the possible existence of real variance via moderator variables. Moderator variables can in simple terms be defined as variables which explain the real variance noticed. Altogether, meta-analysis is able to show how much of the variance can be accounted to statistical errors and how much to the moderator variable(s). It has thus a great potential, although its limitations have to be recognized (see for a critical review Van den Bergh et al., 1997).

\section{Meta-analysis and Value Transfer}

The previous section has set out that a meta-analysis based on regression is able to quantify how much of the variance can be accounted to statistical errors and how much to the moderator variable(s). By means of meta-analysis, it is possible to derive estimations of existing moderator variables, given a well-defined collection of studies. These (parameter-) values may under certain conditions be transferred to other situations.

It is an intriguing question whether transferability of research findings can be considered as an application of meta-analysis. Notwithstanding all research efforts undertaken, in the 
literature an unambiguous distinction between meta-analysis and value transfer has not yet been made. To avoid methodological difficulties we will present a clear distinction here. Metaanalysis uses existing studies as a comparative basis for the study of a particular subject in a more integral way. We define meta-analysis as: a scientific investigation of a well-defined set of previously published individual studies concerning a certain subject, with the aim to apply qualitative as well as quantitative review techniques in order to make an integral crosssectional or comparative study of the available material, leading to more insights on the chosen subject of research and ending up with a systematic synthesis.

Value transfer is closely connected with meta-analysis, but has a different scope. For value transfer we use the following definition: it is a scientific analysis of a subject under study, which aims to use cumulated knowledge generated via previously undertaken similar types of research endeavours in order to draw inferences on hitherto unexplored cases. It serves to meet the formulated study objectives of a repeated study against the least possible research cost.

It should be noticed that our definition is more general than the one of Boyle and Bergstrom (1992) which mentions the transfer of estimated non-market values from the study site towards the policy site. Given our definition of meta-analysis and value transfer, a clear distinction between these two scientific methods can be made. While meta-analysis tries to discover additional information contained in a well-defined collection of studies in order to increase the stock of accumulated knowledge with the aim to arrive at a synthesis, value transfer aims to make use of this form of knowledge accumulation for a new, similar study so as to reach the research objectives at minimum costs. As the next section will show, meta-analysis is one of the methods which generates knowledge suitable for value transfer procedures.

\section{The Basics of Value Transfer}

Recently, quite a few new studies have been presented concerning value transfer. Value transfer, also commonly named benefit transfer, promises the acquisition of the required scientific insights at low costs due to the transfer of relevant information from previously undertaken studies to a new similar type of case study. It has in the past become common practise in the social sciences that even for almost similar problems separate individual studies are initiated in order to derive the insights needed. All such new individual studies incorporate a high research cost. Especially for large research projects these costs are a crucial factor. Hence, it seems rational to investigate whether the application and transfer of a previously undertaken study - when necessary, with slight changes - to a new, similar subject of study is in economic terms - worthwhile. The renewed application of an already developed study might lead to a significant cost saving due to the use of abundant information contained in earlier studies, while, at the same time, the desired research objectives can largely be met.

The name "value transfer" stems from the attempt of transferring (parameter-) values from one case study toward another. The site that serves as origin of the estimated values is called the study site, while the site under consideration is known by the name policy site. Unfortunately, two names are in use to typify the transfer of (parameter-) values from one case study toward another, viz. benefit transfer and value transfer. The use of these two distinct names in 
the scientific literature has caused methodological confusion, a situation often inherent in the development of new research fields. The link between these two identical methods stems, as far as the name is concerned, from the transferability of the (parameter-) values of economic benefit functions from studies undertaken previously toward the new study. Since value transfer - as a name - covers more than benefit transfer, we will use the latter nomenclature.

Value transfers are commonly related to decision-making processes. As illustrated by Button and Kerr (1996), most studies undertaken with the aim of value transfer deal with some form of practically-oriented decision outcomes. According to Loomis (1992), first attempts to carry out transfer of (parameter-) values date back to the 1960s when major research efforts were undertaken on water resources development in the USA. However, not only in decisionmaking processes the use of value transfer can be noticed, but also in modern scientific research a frequent use is made of value transfers. In fact, this approach has already been practised for a long time. However, the name value transfer in connection with this practice is less familiar.Admittedly, value transfer - initially designed to typify the transfer of values of benefit functions - does not cover all possible forms of transfer that can be observed in scientific research. Basically, three levels can be distinguished. First of all, collected data and other relevant information can be transferred. Secondly, the methods used in the study or decisionmaking process can be considered for re-use. And thirdly, the institutional or procedural framework in which the study or decision-making process has taken place can act as guideline for the structure to be used for a new study process, e.g. an investment or research procedure. The analysis concerning structures belonging to the latter approach is mainly carried out in organization-oriented studies and will not be discussed here. However, the transfer of cumulated knowledge and methods will be dealt with in the present section.

Transferring numerical knowledge in scientific investigations (i.e., the first level) was, and still is, common practice. Data sets are used for different studies, parameters derived are used in other studies, and behaviourial elasticities are extensively exchanged (see e.g., Nijkamp and Pepping, 1997). When all required knowledge is present and relevant ceteris paribus conditions are met, value transfer may be permissable. An overview of the scientific literature shows that, in fact, five methods may be distinguished which generate knowledge about the study site(s) that is necessary for the application of value transfer:

(i) a mean value of an endogenous variable is derived on the basis of an analysis of previously undertaken studies;

(ii) a mean value of an endogenous variable is derived (see (i)) and adjusted for possible existing differences in characteristics between the study and the policy site;

(iii) a transfer of demand (or any other behaviourial) function(s) is made (i.e., the trans fer of a function instead of point values);

(iv) random utility models (or other utility based models) are used to assess behaviourial parameters;

(v) meta-analysis based on comparative cross-section may be deployed.

Examples of (i) can be found in Mitchell and Carson (1989). They derive willingness-to-pay (WTP) values by means of direct, on-site, questionnaires. Walsh et al. (1992) assess an average WTP value for swimming, with the aim to transfer this value toward another site. However, there may be many exogenous variables that determine the endogenous WTP value. For the application of (i), the study site must therefore contain characteristics that are highly 
comparable to those of the policy site. Often, many - both small and large - differences exist which make it hard to identify a study (or group of studies) that is almost identical to the policy site. An adaptation for the differences in characteristics may of course ease this limitation.

In case of (ii), the mean value is corrected for the differences in characteristics among the study and the policy site, before it is transferred; see Bergland et al. (1995) for more details.

The third form of value transfer, (iii), transfers the functional relationships and quantities (obtained on the basis of the analysis of the study site) that determine the relevant endogenous variable for the policy site. Loomis (1992) argues that in this case, compared to (i) and (ii), more information is transferred, viz. an entire function. When the exogenous values of the policy site are substituted into this functional form, the value of the endogenous variable follows directly. In contrast to (i) (and in a weaker form for (ii)), none of the studies belonging to the study site are required to be similar in terms of the characteristics of the policy site. In practise, the functional form - which determines the endogenous variable - is a linear or nonlinear combination consisting of the characteristics within the study site.

Next, regarding type (iv), Parsons and Kealy (1994) derive estimators for different characteristics in their random utility model. This model has the feature that it can incorporate substitutes within a decision-making process of a consumer. However, this model does not generate an explicit endogenous quantity, a limitation which makes a value transfer in its original form (i.e. the transfer of concrete (parameter-) values) almost impossible. In fact, in the random utility model, trade-off processes are the subject of transfer. Our definition of value transfer leaves certainly room for this type of transfer.

Finally, (v) requires less restrictions concerning the similarity among the well-defined collection of previous studies, i.e. the study site. By using meta-analysis based on regression, it is possible to derive (parameter-) values as well as more general study characteristics underlying the set of previous studies. Thus, in contrast to the other alternatives, the functional form of impact studies may be derived endogenously. It is worth noticing that a small study undertaken at the policy site can be included in the stock of knowledge of the study site(s). Where relevant, the (parameter-) values can then be adapted to the characteristics underlying the policy site via a Bayesian improvement. This may improve the performance of value transfer, since the knowledge of the policy site is contained in the (parameter-) values.

On the second level, (i.e., of the methods of analysis) value transfer considers the common application of methods among different studies. Although the emphasis in the current literature is mainly on the application of value transfers with regard to supporting decision-making processes, other forms of the use of benefit transfers can be found in practice. Given our definition of value transfer, a transfer is not necessarily limited to the exchange of numerical values. The design of methods and their application generates knowledge which may be applicable elsewhere. Using this cumulated knowledge leads to a reduction of costs (of all kind), given the research objective(s).

Over time, various examples of this second type of value transfer can be observed in scientific research. In the first place, there exists a form of value transfer in which the type of 
method is solely applicable within a particular field of science. This type of transfer can be defined as intra-disciplinary value transfer. An example of this value transfer is the general equilibrium model used in economics. Since the introduction of the general equilibrium model by Arrow and Debreu (1954), it is used to treat a significant share of relevant economic problems. Up till now the general equilibrium model is still a popular tool in economic research. Since this model is used for a broad variety of economic studies, its heterogeneous application can be considered as a form of value transfer.

Secondly, in scientific research also a form of inter-disciplinary value transfer can be found. Here, methods developed in one field of science are used in other fields. Interesting is that a search among different fields of science shows that methods of analysis are used in noncomparable studies. An example of inter-disciplinary value transfers is game theory, which is used in economics to describe the functioning of oligopolistic markets and, at the same time, in evolutionary biology. In a different context, chaos theory is applied in, for example, the study of financial markets (see De Grauwe et al., 1993), as well as in studies about turbulence phenomena in physics (see Ruelle, 1991) and in biological dynamics (see Prigogine and Stengers, 1984) (see for a review Nijkamp and Reggiani, 1997). Similarly, the use of entropy in thermodynamics and in transportation science (Nijkamp and Reggiani, 1994) is an example of inter-disciplinary value transfer. It should be added that such value transfers put a much higher methodological claim on the researcher, as he has to demonstrate also the indigenous relevance and theoretical substance of the concept transferred within his own discipline.

\section{The Theoretical Core of Value Transfer}

Empirically oriented studies suffer from the well-known induction problem, i.e. no observation can be undertaken in a completely context-free way. Every case study - and its findings - is therefore affected by the induction problem. An integral study of a collection of similar studies may mitigate the impact of this type of problem. It is of significant interest to take a closer look at the structure of a constellation (the framework and structure of a phenomenon under study) in which a phenomenon appears, the role of the ceteris paribus condition and the applicability of meta-analysis in this context.

It is important to realize that, generally speaking, knowledge is phenomenon (object or subject)-specific. Knowledge is present and relevant, given the constellation in which the phenomenon exists. The form of appearance of a phenomenon depends on the elements and relationships among these elements. As a result, each constellation as it appears in reality consists of two interconnected components. First of all, there is a physical structure which has the feature that it is observable and measurable. A mutation of this structure will have an effect on the existence of the phenomenon, while the relationships among the physical objects determine what we observe as the phenomenon. Clearly, not only the physical structure determines the constellation; for a constellation a second component is required: an organization. An organization is a pattern (or ordering system) which describes the mutual relationships among elements of the physical structure. This second component is thus the context in which the physical structure must be placed to have a unique meaning. This organization includes the emergence effects and therefore completes the framework which we call constellation. As a consequence, the constellation describes a unique environment. A phenom- 
enon that appears in this environment is therefore unique too. It is in this context now interesting to take a more formal look at the structure leading to new knowledge. Although this breakdown (commonly called analysis) increases the objectivity (i.e. the measurability) of the elements, the emergence effects are then neglected. An illustrative example of emergence effects is the taste of sugar which cannot be determined by its particles solely; see Capra (1997).

We will now offer a formal framework for value transfer studies. As a start of our analysis, the relationship between a set of all observable objects and a set of properties (attributes) that describe a certain object will be investigated. Intuitively, it is clear that the more precise we define the set of properties, the lower the number of objects that will satisfy all these properties. At the same time, the larger the set of properties the more knowledge we have accumulated on the remaining set of objects. It can be proven that, given a well-defined collection of objects, the more attributes are necessary to describe a constellation, the more specific the knowledge concerning this constellation becomes. In scientific investigations, under three assumptions an artificial constellation - meant to model the constellation as it appears in reality - can be built. In this context, we assume in the first place that it is possible to distinguish (physical) elements (which means that a certain degree of isolation is possible); secondly, that relationships are unique; and finally that the artificial constellation we consider can be described by a finite set of attributes and relationships. Given elementary set theory, we can now prove that an additional property reduces the size of the set of elements that satisfies all properties.

\section{Proof}

Let $\mathrm{x}$ describe an observable phenomenon existing in the real world, $\mathrm{P}_{\mathrm{i}}$ the property or attribute, and $\left\{x \mid P_{i}(x)\right\}$ the set of objects satisfying this property. The set of properties $(\mathrm{i}=1, \ldots, \mathrm{n})$ can be written as

$$
\left\{P_{i}(x) \mid \bigcup_{i=1}^{n} P_{i}(x)\right\}
$$

If $\mathrm{i}$ is the index set such that $\mathrm{i} \in \mathbb{N}$, we can write three basic rules of set theory as follows:

$$
\begin{aligned}
& \left\{x \mid P_{i}(x)\right\} \cap \varnothing=\left\{x \mid P_{i}(x)\right\} \quad \forall i \\
& \left\{x \mid P_{i}(x)\right\} \cap\left\{x \mid P_{i}(x)\right\}=\left\{x \mid P_{i}(x)\right\} \forall i
\end{aligned}
$$

and

$$
\left\{x \mid P_{i}(x)\right\} \cap\left\{x \mid P_{j}(x)\right\}=\left\{x \mid P_{j}(x)\right\} \cap\{x \mid j
$$

Now, given the index set $\mathrm{i}$, and by the assumption that $\mathrm{m}, \mathrm{n} \in \mathrm{i} \wedge 1 \leq \mathrm{m}<\mathrm{n}$, we may write

$$
\left\{x \mid \bigcap_{i=1}^{m} P_{i}(x)\right\} \cap\left\{x \mid \bigcap_{i=n-m}^{n} P_{i}(x)\right\}=\left\{x \mid \bigcap_{i=1}^{n} P_{i}(x)\right\}
$$

When the set of properties contains non-trivial elements, this means that 


$$
\left\{x \mid \bigcap_{i=n-m}^{n} P_{i}(x)\right\} \neq \varnothing \wedge\left\{x \mid \bigcap_{i=n-m}^{n} P_{i}(x)\right\} \neq\left\{x \mid \bigcap_{i=1}^{m}\right.
$$

from which follows that

$$
\left\{x \mid \bigcap_{i=1}^{m} P_{i}(x)\right\}>\left\{x \mid \bigcap_{i=1}^{n} P_{i}(x)\right\}
$$

Thus, an additional (group) of non-trivial properties causes a reduction in the amount of objects contained in the set satisfying all properties. Q.E.D.

For our proof concerning the mutual size of the set of properties and the objects satisfying all properties, we made use of the feature that the set of properties did not contain trivial properties. We will now show that this is a redundant assumption.

\section{Proof}

Given (2) it can be easily derived that imposing a property more than once has no effect on the size of the set containing the elements satisfying the set of properties.

\section{Q.E.D.}

Next, we argue that a property can be classified as trivial, if it does not affect the set of objects that satisfies the set of properties. This can be demonstrated as follows.

\section{Proof}

An intersection of the set of properties consisting of $m$ properties with another property $m+1$ such that

$$
\left\{x \mid \bigcap_{i=1}^{m} P_{i}(x)\right\} \cap\left\{x \mid P_{m+1}(x)\right\}=\varnothing
$$

implies that the property $\mathrm{P}_{\mathrm{m}+1}(\mathrm{x})$ will not be an element of the set of properties. As a consequence, a property is relevant if it satisfies the following condition:

$$
\left\{x \mid \bigcap_{i=1}^{m} P_{i}(x)\right\} \cap\left\{x \mid P_{m+1}(x)\right\} \neq \varnothing \Rightarrow P_{m+1} \notin\{P \text {. }
$$

\section{Q.E.D.}

Next, we can also demonstrate that the ordering within the set of properties plays no role in the description of the entire set of properties.

\section{Proof}

Since (4) can be applied for all elements of

$$
\left\{x \mid \bigcap_{i=1}^{n} P_{i}(x)\right\}
$$

it is easily seen that an ordering within the set of properties has no implications for the set resulting from the complete set of properties. Q.E.D. 
From the previous proofs it can be deduced that an additional property leads to a reduction of the set of elements and to an increase of specific knowledge due to the enlargement of the set of properties (see subsequent proof). Hence, there exists a pay-off between the size of the set of properties and the specificity of knowledge. When another property induces an empty set of phenomena at the next stage, a more detailed description of the set of phenomena satisfying the set of properties is not possible.

That the constellation is unique, given the set of properties, is easy to derive. When we compare two sets of properties, it is straightforward that

$$
\left\{x \mid \bigcap_{j=1}^{m} P_{j}(x)\right\} \neq\left\{x \mid \bigcap_{i=1}^{n} P_{i}(x)\right\},
$$

in case $m=n$ and at least one property is different between both sets of properties; or (11) holds when the set of properties differ in size, i.e. $n \neq m$. Now we offer the proof of this statement.

\section{Proof}

Assume that

$$
\left\{P_{i}(x) \mid \bigcup_{i=1}^{n} P_{i}(x)\right\}=\left\{P_{j}(x) \mid \bigcup_{j=1}^{m} P_{j}(x)\right\}
$$

This implies that both sets contain the same quantity of properties. Another implication is that $\mathrm{P}_{\mathrm{i}}(\mathrm{x})=\mathrm{P}_{\mathrm{j}}(\mathrm{x}) \forall \mathrm{i}=\mathrm{j}$. If $\exists \mathrm{P}_{\mathrm{i}}(\mathrm{x}) \neq \mathrm{P}_{\mathrm{j}}(\mathrm{x})$ given that $\mathrm{m}=\mathrm{n}$, equation (12) is no longer valid. This can also be used for the case in which $m \neq n$. Let $P_{i}^{m}$ and $P_{j}^{m}$ be the properties belonging to the index set $\mathrm{i}$, respectively $\mathrm{j}$, at $\mathrm{m}$. Then, these two properties can only be equal if

$$
P_{j}^{m}(x)=P_{i}^{m}(x)
$$

When $\mathrm{m}=\mathrm{n}, \mathrm{P}_{\mathrm{i}}^{\mathrm{m}}$ and $\mathrm{P}_{\mathrm{j}}^{\mathrm{m}}$ are single properties. In case $\mathrm{m}<\mathrm{n}$, the next equation must be

$$
P_{j}^{m}(x)=P_{i}^{m}(x)=\bigcap_{i=n-m-1}^{n} P_{i}(x),
$$

i.e. $\mathrm{P}_{\mathrm{i}}^{\mathrm{m}}$ is now treated as an intersection of properties which is compared with the property of $\mathrm{P}_{\mathrm{j}}$ at $\mathrm{m}$. This is useful, since the remaining properties of the index set $\mathrm{i}$ from $\mathrm{n}-\mathrm{m}-1$ may represent a subsystem equal to the property $P_{j}^{m}$. This would imply that (12) would hold. However, when the properties of $i$ over $\{n-m-1, . ., n\} \subset \mathbb{N}$ do not describe a subsystem resulting in $\mathrm{P}_{\mathrm{j}}^{\mathrm{m}}$ or when $\mathrm{P}_{\mathrm{i}}(\mathrm{x})$ does not consist of a repetition of $\mathrm{P}_{\mathrm{i}}^{\mathrm{m}}$, then if $\exists \mathrm{P}_{\mathrm{i}}(\mathrm{x}) \in \mathrm{P}_{\mathrm{i}}^{\mathrm{m}}$ which is unequal to $\mathrm{P}_{\mathrm{j}}(\mathrm{x})$, it follows that $\mathrm{P}_{\mathrm{i}}^{\mathrm{m}}(\mathrm{x}) \neq \mathrm{P}_{\mathrm{j}}{ }^{\mathrm{m}}(\mathrm{x})$. As a consequence, we have to reject (12). So the two sets of properties consist of different elements and are thus each spanning a constellation which differs from each other. Q.E.D.

As a result of our way of modelling constellations, it is clear that differences between phenomena are induced by differences in the composition of the set of properties (which describes the constellation in which a certain phenomenon exists). It has to be noticed that a property (or a set of attributes) of an object can comprise more than simple static attributes. We can add space as a property or dimension to the set of properties. This property is a 
relationship based on a discrete or continuous function. Furthermore, especially the addition of time to the set of properties has significant implications. Time is an unique ordering measure of events, as it is irreversible. As Prigogine and Stengers (1984) point out, its explicit justification lies in irreversible processes that can be observed in real-life, for example, chemical processes. A direct consequence is that the set of properties which include time is a set of properties which spans an abstract constellation which is unique for each moment in time. This makes data concerning (groups of) properties time-specific and, therefore, possibly less useful for value transfer. Nevertheless, in reality correction mechanisms are available to adjust knowledge to changes over time. For example, in economics real values are used to outweigh changes in monetary variables over time. A similar remark can be made on the properties representing the dimension of space or the combined dimension space and time. This last mentioned property is in fact a combination of the properties time and space. As the last proof already indicates, our mathematical formulation of a constellation leaves room for the inclusion of a combination of properties to describe a subsystem of this constellation, since a single property may represent a subsystem as such. We can simply substitute $\mathrm{P}_{\mathrm{i}}(\mathrm{x})$ for the intersection

$$
P_{\text {time }}(x) \cap P_{\text {space }}(x)
$$

or for any other set of intersections. It is often forgotten is that an (imposed) assumption underlying a theory or analysis is a property too, because its imposition adds an additional piece of knowledge, fact or limitation to the analysis which considers a well-defined collection of properties that, on a final level, spans the constellation.

After the formal presentation of the above proofs, we will now trace the implications for value transfer. The more specific a body of knowledge gets, the less likely it is that the cumulated knowledge can be used somewhere else. Furthermore, analytical and financial limitations often reduce the ability of handling such a large body of knowledge. However, if a dis-connection of the total stock of cumulated knowledge is possible, parts that satisfy a group of properties may be used for value transfer. By definition, a split-up among subsystems induces a loss of information, i.e. knowledge. However, each subsystem may contain knowledge that may be transferred. A nice example of subsystems are engine management systems, built in cars with the aim to optimize engine performance (for example, to reduce air pollution). Also in economic analysis several subsystems can be distinguished, for instance, in transport economics. Electronic traffic guidance based on telematics is a subsystem meant to optimize the use of infrastructure aiming to reduce the (economic) cost, for example, induced by congestion and accidents.

We may also refer here to the ceteris paribus condition, which is a powerful tool in economic analysis to focus on one subset in our investigations. Nevertheless, important is to realize which properties are affected by the ceteris paribus condition (see also Rivett, 1970). It is clear that properties containing information which is heavily depending on area- and timespecific factors will have a larger impact than those which represent more general aspects of a phenomenon. The consequences for the performance with respect to value transfer of subsets is in this context clear. Nevertheless, in various cases we can globally correct for contextdepending knowledge. For example, Smith and Osborne (1996) consider a test to judge the internal consistency of $\mathrm{CV}$ value estimates. By means of the application of meta-analysis they were able to take into account a distinct valuation of air pollution by inhabitants from the East 
and West part of the US for an improvement of the visibility at national parks. It is noteworthy that such methods are approximations for incorporating real-life changes into the model. Such changes can be observed, approximated and brought into an analytical framework, but the causes of change are largely neglected. However, changes in real-life often contain specific information that may be useable for comparative studies, such as meta-analysis. Meta-analysis is in principle able to extract certainly additional knowledge from a body of similar, but independent (case-) studies.

Another implication of our mathematical treatment of the description of a phenomenon is straightforward. The more complex the constellation observed gets, the more effort is required to distil all relevant knowledge. Hence, a phenomenon as we observe in a more complex constellation is created by a larger set of properties. In fact, multiple properties determine a certain phenomenon. To describe this more complex phenomenon with the aim to end up with a theory, requires more research efforts - in the sense of observation, analysis and synthesis which will induce a higher cost. The application of meta-analysis and value transfer seems beneficial in this context.

In conclusion, the larger the set of properties which spans the constellation in which a phenomenon does exist, the smaller the set of elements that satisfy these properties. This inverse relationship shows that - due to different types of limitations - only parts of the cumulated knowledge can be transferred to other phenomena to describe their constellation. When research findings are not context-free, the generalization possibilities of cumulated knowledge via value transfer is limited.

\section{Value Transfer: Ceteris Paribus Condition and Performance}

By definition, a scientific analysis will focus on simple (or simplified) properties of a phenomenon which explain its existence. This is a selective process which is influenced by data availability, financial constraints, existing methodologies, prior assumptions, the theoretical framework adopted etc.. Consequent, a similar phenomenon investigated in a sequence of independently undertaken studies may be treated from different perspectives. Value transfer aims to offer a systematic framework for this question. Using the jargon from economics, we may say that value transfer is a sophisticated method for dealing with ceteris paribus conditions. We assume essentially that some properties of a phenomenon have a more significant impact than others which may thus be assumed to be constant. Clearly, only a small part of site-specific differences among studies are analyzed and accepted as explanatory variables. This may have an influence on the performance of value transfer. For these particular site-specific differences between the study site and the policy site, an adjustment can be made to derive the adjusted (parameter-) values for the new study, i.e. the policy site. In this context the other effects, which belonged to those satisfying the ceteris paribus condition, are taken over without any adjustment. Consequently, for all studies of the well-defined set of previous studies, the variables satisfying the ceteris paribus condition must be the same. For example, in a nonlinear dynamic system the constellation may exhibit complex behaviour, so that stable values may be more problematic; otherwise, when not all variables are properly taken into account, this will finally affect the accuracy of the derived (parameter-) values. It is worth noting that meta-analysis is the only method currently available which has the feature that it is 
able to discover (depending on the format of data and the technique used) explanatory (i.e. moderator) variables underlying the well-defined collection of studies. Meta-analysis in general is able to isolate the explanatory variables from those from which we can abstract, and so may serve as a tool for the search of those variables which satisfy the ceteris paribus condition.

Beside earlier mentioned features of finding statistical errors and synthesing, meta-analysis can be expected to have the ability to determine the borders of a subset of accumulated knowledge. It can sort out non-relevant variables; variables which satisfy the ceteris paribus condition; and finally explanatory variables. In the previous section we have pointed out that a subset of cumulated knowledge seems to be more proper for the application of value transfer. Returning to the ceteris paribus condition, especially Bergland et al. (1995) have made a serious attempt to approach a situation of ceteris paribus conditions in their study. Thereto they have undertaken a direct test of the value transfer approach by conducting two parallel and similar CV studies concerning water quality. Remarkable is, however, that even then value transfer does not show up as a promising method. Thus, due to the rich environment of influencing forces the ceteris paribus condition is not likely to hold when such analyses are used for cases found in reality. Additionally, the ceteris paribus condition implies that the average benefit from an activity (or the parameters of the benefit function) at the policy site are those values estimated, given the knowledge accumulated from the study site(s). A successful application of benefit function transfer requires therefore the equality of parameters of the study site and the policy site. Interestingly enough, in a cross-state study concerning the fishery sector, undertaken by Loomis (1992), the null hypothesis of the existence of equality of coefficients between two sites was rejected, not only for the average benefit per activity but also for the benefit functions. But also from another point of view the results of Loomis are interesting. A successful application of benefit function transfer requires therefore the equality of parameters of the study site and the policy site. Hence, the implicit assumption which legitimizes value transfers is that (parameter-) values derived at a certain point in time or on one location for a particular decision-making process or study can be deployed in other equivalent decisionmaking procedures or studies. Basically, value transfer relies therefore on the "global" predictive power of the initial developed model (where global is meant in the sense of stability). This stability condition means converging toward a real value in the limit, i.e. there is a tendency toward the optimum: with every iteration an adjustment closer to the optimum takes place. Thus, the dynamic behaviour tends to satisfy Cauchy's principle of convergence. Only fixed points will be mapped on their initial positions and thus the path of adjustment describes a dynamic process; see for more details for example Stokey et al. (1989). From this point of view, the position along these trajectories for mutual independent identical systems determine the performance of value transfer.

\section{Conclusion}

In this paper a methodological quantitative economic analysis is presented to yield new insights into value transfer (also known as benefit transfer) as a tool of research. Within this research perspective, we were able to get a better understanding of the working of this approach and to derive a clear distinction between meta-analysis and value transfer, an issue which has not been discussed thoroughly before. Additionally, given the new formulated definitions we could distinguish different types of value transfer and were able to clarify existing methodological confusion concerning value transfer. 
Via a quantitative analysis we were able to point out that only subsets of an entire property set are likely subject to transfer. Furthermore, we were able to indicate the relationship between the ceteris paribus condition and meta-analysis with respect to value transfer. For example, via a meta-regression it can be determined which variables will satisfy the ceteris paribus condition and which are explanatory ones, provided the external environment allows to make stability assumptions. In our approach we argued that via meta-analytic techniques it may be possible to discover the borders of a subset of accumulated knowledge, given a phenomenon under study. As a consequence, these quantitative techniques may serve as a tool to determine existing relevant subsets in a body of knowledge, a feature important for value transfer applications. In the light of our results, also a renewed discussion concerning the ceteris paribus condition seems to be warranted.

\section{References}

Arrow, K.J. and Debreu, G. (1954) Existence of an Equilibrium for a Competitive Economy, Econometrica, vol. 22, pp. 265-290.

Bateman, I.J., Brainard, J.S. and Lovett, A.A. (1995) Modelling Woodland Recreation Demand Using Geographical Information Systems: A Benefit Transfer Study, CSERGE Working Paper GEC 95-06, University of East Anglia, Norwich.

Bergh, J.C.J.M. van den, and Button, K.J. (1997) Meta-analysis of Environmental Issues in Regional, Urban and Transport Economics, Urban Studies, vol. 34, Nos 5-6, pp. 927-944.

Bergh, J.C.J.M. van den, Button, K.J., Nijkamp, P. and Pepping, G.C. (1997) Meta-analysis in Environmental Economics, Kluwer Academic Publishers, Dordrecht.

Bergland, O., Magnussen, K. and Navrud, S. (1995) Benefit Transfer: Testing for Accuracy and Reliability, Discussion Paper \#D-03/1995, Agricultural University of Norway, Oslo.

Boyle, K.J. and Bergstrom, J.C. (1992) Benefit Transfer Studies: Myths, Pragmatism, and Idealism, Water Resources Research, vol. 28, pp. 657-663.

Button, K.J. and Kerr, J. (1996) Effectiveness of Traffic Restraint Policies: a Simple Metaregression Analysis, International Journal of Transport Economics, vol. 23, pp. 214-225.

Button, K.J. and Rietveld, P. (1997) Infrastructure Policy and Pan-European Regional Development, in H. Kohno, P. Nijkamp and J. Poot (eds.), Regional Cohesion and Competition in the Process of Globalization, Edward Elgar, Cheltenham (forthcoming).

Capra, F. (1997) Web of Life: A New Synthesis of Mind \& Matter, Flamingo, London.

Carson, R.T., Flores, N.E. Martin, K.M. and Wright, J.L. (1996) Contingent Valuation and Revealed Preference Methodologies: Comparing the Estimates for Quasi-public Goods, Land Economics, vol. 72, pp. 80-99. 
Gowdy, J.M. (1997) The Value of Biodiversity: Markets, Society, and Ecosystems, Land Economics, vol. 73, 1, pp. 25-41.

Grauwe, P. de, Wachter, H. de, and Embrechts, M. (1993) Exchange Rate Theory: Chaotic Models of Foreign Exchange Markets, Blackwell, Oxford.

Hunter, J.E. and Schmidt, F.L. (1990) Methods of Meta-analysis, Sage Publications, London.

Kirchhoff, S. Colby, B.G. and LaFrance, J.Y. (1997) Evaluating the Performance of Benefit Transfer: An Empirical Inquiry, Journal of Environmental Economics and Management, vol. 33, pp. 75-93.

Loomis, J.B. (1992) The Evolution of a More Rigorous Approach to Benefit Transfer: Benefit Function Transfer, Water Resources Research, vol. 28, 3, pp. 701-705.

Matarazzo, B. and Nijkamp, P. (1997) Meta-analysis for Comparative Environmental Case Studies: Methodological Issues, International Journal of Social Economics, vol. 24, pp. 799811.

Mitchell, R. and Carson, R. (1989) Using Surveys to Value Public Goods: The Contingent Valuation Method, Resources for the Future, Washington D.C.

Nijkamp, P. and Pepping, G.C. (1997), A Meta-approach to Investigate the Variance in Transport Price Elasticities: a Cross-national European Comparison, International Journal of Transport Statistics (forthcoming).

Nijkamp, P. and Reggiani, A. (1994) Interaction, Evolution and Chaos in Space, SpringerVerlag, Berlin.

Nijkamp, P. and Reggiani, A. (1997) The Economics of Complex Spatial Systems, Elsevier, Amsterdam (forthcoming).

Parsons, G.R. and Kealy, M.J. (1994) Benefits Transfer in a Random Utility Model of Recreation, Water Resources Research, vol. 30, 8, pp. 2477-2484.

Prigogine, I. and Stengers, I. (1984) Order Out of Chaos, Bantam Books, New York.

Rivett, K. (1970) "Suggest" or "Entail"? The Derivation and Confirmation of Economic Hypotheses, Australian Economic Papers, pp. 127-146.

Ruelle, D. (1991) Chance and Chaos, Princeton University Press, Princeton.

Sagoff, M. (1988) The Economy of the Earth, Cambridge University Press, Cambridge.

Smith, V.K. and Osborne, L. (1996) Do Contingent Valuation Estimates Pass a 'Scope' Test? A Meta Analysis, Journal of Environmental Economics and Management, vol. 31, pp. 287301. 
Stokey, N., Lucas Jr., R.E. and Prescott, E.C. (1989) Recursive Methods in Economic Dynamics, Harvard University Press, Cambridge.

Tombe, D.J. de (1994) Defining Complex Interdisciplinary Societal Problems: a Theoretical Study for Constructing a Comparative Problem Analyzing Method, Dissertation Thesis, University of Amsterdam, Amsterdam.

Walsh, R.G., Johnson, D.M. and KcKean, J.R. (1992) Benefit Transfer of Outdoor Recreation Demand Studies, 1968-1988, Water Resources Research, vol. 28, 3, pp. 707-713.

Waters, W.G. (1995) Values of Travel Time Savings in Road Transport Project Evaluation, Paper $7^{\text {th }}$ World Conference of Transport Research, Sydney. 